\title{
A NOTE ON CONSTRUCTIBLE LATTICES
}

\author{
D. H. ADAMS \\ (Received 11 May 1971; revised 30 July 1971) \\ Communicated by P. D. Finch
}

It is our purpose to show that the constructible orthomodular lattices defined by Janowitz in [2], are embeddable into Boolean lattices. In fact they are subdirect products of Boolean lattices, where the subdirect products are taken in the class of orthomodular posets. We shall make these notions precise. Other concepts, such as disjoint sum and constructible lattice, are defined in [1] and [2].

Definition 1. If $L_{1}$ and $L_{2}$ are two orthomodular posets, then a map $\phi$ : $L_{1} \rightarrow L_{2}$ is called a homomorphism if, for all $x$ and $y$ in $L_{1}$,

If, in addition

(i) $\phi\left(x^{\perp}\right)=\phi(x)^{\perp}, \phi(0)=0$;

(ii) $x \perp y$ implies that $\phi(x \vee y)=\phi(x) \vee \phi(y)$.

(iii) $x \leqq y$ if and only if $\phi(x) \leqq \phi(y)$

then $\phi$ is called an embedding.

Note that this definition is stronger than Definition 1.4 of Zierler and Schlessinger [4], but that it is the same as the notion used by Kochen and Specker [3].

Definition 2. Let $\left\{L_{\alpha}: \alpha \in A\right\}$ be a family of orthomodular posets, let $\Pi\left\{L_{\alpha}: \alpha \in A\right\}$ denote the direct product of this family and let $i_{\alpha}: \Pi\left\{L_{\alpha}: \alpha \in A\right\} \rightarrow L_{\alpha}$ denote the natural projection of the direct product onto its component $L_{\alpha}$. Then an orthomodular poset $L$ is called a subdirect product of the family $\left\{L_{\alpha}: \alpha \in A\right\}$ if

(i) there is an embedding $\phi: L \rightarrow \Pi\left\{L_{\alpha}: \alpha \in A\right\}$;

(ii) $i_{\alpha}$, restricted to the range of $\phi$, is still onto $L_{\alpha}$.

THEOREM 1. Let $\left\{L_{\alpha}: \alpha \in A\right\}$ be a family of orthomodular posets such that, for any $\alpha$ in $A$, there exists a homomorphism $h_{\alpha}$ of $L_{\alpha}$ onto 2 , the two-element Boolean lattice. Then $L=D S\left\{L_{\alpha}: \alpha \in A\right\}$, the disjoint sum of the family $\left\{L_{\alpha}\right.$ : $\alpha \in A\}$, is a subdirect product of $\left\{L_{\alpha}: \alpha \in A\right\}$.

Proof. We define an embedding $\phi$ of $L$ into $\Pi\left\{L_{\alpha}: \alpha \in A\right\}$.

Suppose $a \in L$; if $a=0,1$ we put $\phi(a)=0,1$ in $\Pi\left\{L_{x}: \alpha \in A\right\}$ respectively. 
If $a \neq 0,1$ then it belongs to a unique $L_{\alpha}$. If $h_{\alpha}(a)=0$, we define $\phi(a)$ as a function on $A$ by, if $\beta \in A$,

$$
\phi(a)(\beta)= \begin{cases}0_{\beta} & (\beta \neq \alpha), \\ a & (\beta=\alpha) .\end{cases}
$$

If, on the other hand, $h_{\alpha}(a)=1$ then we define $\phi(a)$ by

$$
\phi(a)(\beta)= \begin{cases}1_{\beta} & (\beta \neq \alpha), \\ a & (\beta=\alpha) .\end{cases}
$$

It is routine to verify that $\phi$ is an embedding, and the the natural projections onto the components of the direct product are still onto when restricted to the range of $\phi$.

The next theorem is our main result.

THEOREM 2. A constructible lattice is a subdirect product of the Boolean lattices used to construct it.

Proof. The theorem is trivial for 0-constructible lattices as they are Boolean. We proceed by induction on the degree of constructibility: suppose the theorem true for $i$-constructible lattices where $i<2 n$.

First we observe that a subdirect product $L$ of the subdirect products $\left\{L_{\alpha}\right.$ : $\alpha \in A\}$ is clearly a subdirect product of the components of the $L_{\alpha}$. The direct product is obviously a subdirect product, hence the theorem is true for $(2 n)$-constructible lattices. A subdirect product of Boolean lattices admits plenty of homomorphisms onto 2 , hence the theorem is true for $(2 n+1)$-constructible lattices by Theorem 1 . The proof is complete.

Corollary 3. A constructible lattice is embeddable into a Boolean lattice. Proof. The direct product of Boolean lattices is Boolean.

\section{References}

[1] M. F. Janowitz, 'The near center of an orthomodular lattice', J. Aust. Math. Soc.,XIV (1972), 20-29.

[2] M. F. Janowitz, 'Constructible Lattices', J. Aust. Math. Soc., XIV (1972), 311-316.

[3] S. Kochen and E. P. Specker, 'The problem of hidden variables in quantum mechanics', $J$. Math. Mech. 17 (1967), 59-87.

[4] N. Zeirler and M. Schlessinger, 'Boolean embeddings of orthomodular sets and quantum logic'. Duke Math. J. 32 (1965), 251-262.

Monash University

Clayton, Victoria, Australia

Present address

University of Massachusetts

Amherst, Massachusetts, 01002

U.S.A. 